\section{Kidney \\ Blood Pressure Research}

\title{
Kidney Function and Cognitive Impairment in People Aged 80 Years and Over with Untreated Hypertension: A Cross-Sectional Survey
}

\author{
Han Wang ${ }^{a}$ Tao Liu ${ }^{b}$ Ying-ying Caic Hui Jiang ${ }^{a}$ Han-xiong Liu ${ }^{a}$ Cai Lin ${ }^{a}$ \\ ${ }^{a}$ Cardiovascular Disease Research Institute, The Third People's Hospital of Chengdu, The Second \\ Affiliated Chengdu Clinical College of Chongqing Medical University, Chengdu, Sichuan, bepartment \\ of Neurology, People's Hospital of Hainan Province, Haikou, Hainan, 'Department of Geriatric, The First \\ Affiliated Hospital of Chengdu Medical College, Chengdu, Sichuan, China
}

\section{Key Words}

Chronic kidney disease $\cdot$ Cognitive impairment $•$ Hypertension $•$ Aged

\begin{abstract}
Background/Aims: Both kidney dysfunction and cognitive impairment are common problems in hypertensive patients. However, few studies have explored the association between these conditions in hypertensive patients aged 80 or over. The current study was undertaken to determine the impact of chronic kidney disease (CKD) and estimated glomerular filtration rate (eGFR) on cognitive impairment among an 80-year-old population with untreated hypertension in China. Methods: A total of 395 hypertensive patients aged 80 or over were assessed for the presence of cognitive impairment according to the 30-item Mini-Mental State Examination (MMSE). Cognitive impairment was defined as a score below 24 on MMSE. eGFR was evaluated using the Chinese Modification of Diet in Renal Disease equation. CKD was defined according to categorical approach, which is based on "YES" (eGFR below $60 \mathrm{ml} / \mathrm{min}$ ) or "NO" (eGFR above $60 \mathrm{ml} / \mathrm{min}$ ). Results: The mean (SD) age was $83.0 \pm 2.6$ years for the sample, of whom $69.8 \%$ were female. There were 59 (14.9\%) and 280 (71.1\%) prevalent cases of CKD and cognitive impairment, respectively. CKD patients were older, had higher scores on Activity of Daily Living (ADL), and lower score on MMSE. After controlling for potential confounding, multiple logistic regressions demonstrated that both CKD and eGFR were associated with cognitive impairment in hypertensive patients aged 80 or over. Conclusion: Our study found that both CKD and eGFR were associated with cognitive impairment among hypertensive patients aged 80 or over in China. Therefore, targeted screening for cognitive impairment should be considered in these patients with CKD.
\end{abstract}

Copyright @ 2016 S. Karger AG, Basel

H. Wang and T. Liu contributed equally to this article and therefore share first authorship.

Prof. Cai Lin

KARGER 125\%
Cardiovascular Disease Research Institute, The Third People's Hospital of Chengdu, The Second Affiliated Chengdu Clinical College of Chongqing Medical University, Chengdu, Sichuan 610031, P.R.China. 82 Qinlong St. Chengdu, Sichuan (China)

E-Mailwh7967@hotmail.com 


\section{Kidney Blood Pressure Research}

Kidney Blood Press Res 2016;41:70-77

\begin{tabular}{l|l}
\hline DOI: 10.1159/000368550 & (C) 2016 S. Karger AG, Basel
\end{tabular}

Published online: February 14, 2016

www.karger.com/kbr

Wang/Liu/Cai/Jiang/Liu/Lin: Kidney Function and Cognitive Impairment with Untreated Hypertension in Elderly

\section{Introduction}

Both kidney dysfunction and cognitive impairment are common problems in elderly people which have severe consequences, such as diminished quality of life, higher mortality rate, and increased use of health services[1,2]. Currently, $10 \%$ of the world's population is aged over 60 , and the very old people, aged over 80 , are the fastest growing group of older people today [3]. The ageing of population along with the growing global prevalence of hypertension has also led to a corresponding worldwide increase in the prevalence of chronic kidney disease (CKD) and cognitive impairment $[4,5]$.

Recent cross-sectional and prospective studies have suggested that decreased kidney function is associated with cognitive impairment, cognitive decline and dementia in the general population, and diabetes mellitus [6-11]. These authors explained that high risk of cardiovascular and cerebrovascular diseases can increase risk cognitive impairment in patients with impaired kidney function[6-11]. Additionally, many conditions such as anemia, hypoproteinemia, and oxidative stress, may link CKD with reduced cognitive function $[12,13]$. Furthermore, accumulative evidence has also demonstrated that the association remains unchanged even after controlling for cardiovascular and cerebrovascular diseases, suggesting that reduced kidney function may be an independent risk factor for cognitive impairment, although their mechanism is still unclear. However, results from other studies have also provided compelling evidence [14].

A common feature in these studies is the relatively young age of the participants. These risk factors for cognitive impairment may differ in the very elderly [3]. In fact, mortality in the elderly might also differ among different age groups, thus removing a correlation between CKD and cognitive impairment. For example, authors found that depression was not directly associated with cognitive impairment in Chinese nonagenarians and centenarians [15]. In addition, reduced kidney function and cognitive impairment are also common problems in hypertensive patients $[5,11]$. The Hypertension in the Very Elderly Trial showed significant association between cognitive function/dementia and serum creatinine in hypertensive persons aged 80 or over $[3,16]$. Few studies, however have explored the association between kidney function and cognitive impairment in untreated hypertensive patients aged 80 or over, which may further demonstrate the relationship between the two conditions. The current study was undertaken to determine the impact of chronic kidney disease (CKD) and estimated glomerular filtration rate (eGFR) on cognitive impairment among untreated hypertensive persons aged 80 or over in China.

\section{Materials and Methods}

\section{Study subjects}

The study population was selected by the cluster sampling method according to the database supplied by the Hypertension and Ageing Project of Chengdu (HAPC). The HAPC was performed in Chengdu city in order to explore the association among chronic cardiovascular diseases in rural inhabitants. In September 2007, rural inhabitants of Chengdu aged 80 or over were invited to join the study by oral notification. Out of 8461 subjects, 1423 responded to the invitation and agreed to participate in the study. Trained personnel visited all study participants at their homes for data collection, biological specimen collection, and anthropometric measurements. The current study is a part of the HAPC, and its purpose is to assess the association between the kidney function and cognitive impairment in the 80-year-old population with untreated hypertension. All patients with untreated hypertension were selected in the population. Patients with untreated hypertension were defined as a subject with hypertension who had never received antihypertensive drugs, or had not taken appropriate medication for at least three months. The current study ultimately included 395 rural inhabitants with untreated hypertension. The study was approved by the ethic committee of the Third People's Hospital of Chengdu. Written informed consent was obtained from subjects enrolled. 


\section{Kidney \\ Blood Pressure Research}

Kidney Blood Press Res 2016;41:70-77

\begin{tabular}{l|l}
\hline DOI: 10.1159/000368550 & (C) 2016 S. Karger AG, Basel
\end{tabular}

Published online: February 14, 2016

www.karger.com/kbr

Wang/Liu/Cai/Jiang/Liu/Lin: Kidney Function and Cognitive Impairment with Untreated Hypertension in Elderly

\section{Estimation of kidney function}

Blood sample for measurement of serum creatinine was obtained at baseline after an overnight fast. Serum creatinine was measured on frozen serum at a central laboratory using the Kodak Ektachem 700 Analyzer (Biochemistry laboratory, The Third Hospital of Chengdu). Estimated glomerular filtration rate (eGFR) was assessed using the Chinese Modification of Diet in Renal Disease equation, which is based on baseline age, gender, and serum creatinine concentration [17]. CKD was defined according to categorical approach, which is based on "YES" (eGFR below $60 \mathrm{ml} / \mathrm{min}$ ) or "NO" (eGFR above $60 \mathrm{ml} / \mathrm{min}$ ).

\section{Cognitive impairment testing}

Chinese adapted MMSE (CMMSE) has been utilized as a dementia screening instrument for epidemiological studies, and minimally modified culturally and formally to keep it in line with the original [18]. The CMMSE was used to assess cognitive function. It categorizes subjects according to the following: cognitive impairment (scores of 0-18), mild cognitive impairment (scores of 19-24), and normal cognitive functions (scores of 25-30). The MMSE scores have been shown to be $80 \%-90 \%$ sensitive and $80 \%-100 \%$ specific for the diagnosis of cognitive impairment [19]. The scoring system was used to assess the cognitive function of elderly Chinese in rural districts and demonstrated similar sensitivity and specificity [20].

\section{Measurements of other covariates}

Other covariates included sociodemographic characteristics (age, gender, education, live alone, and smoking), relevant medical history (heart disease, stroke and diabetes), score on ADL, anthropometric measurement (body mass index) and biochemistry indicators (hemoglobin, albumin, triacylglycerol, total cholesterol, low-density lipoprotein cholesterol and high-density lipoprotein cholesterol). Sociodemographic characteristics were collected on the basis of the prepared questionnaire for the sample. Sitting or recumbent position, right arm blood pressure (BP) was measured twice to the nearest $2 \mathrm{mmHg}$ using a standard mercury sphygmomanometer (phases I and V of Korotkoff) by trained nurses or physicians. The mean value of two measurements was used to calculate systolic BP (SBP) and diastolic BP (DBP). Diagnosis of hypertension was confirmed according to the criteria of the Joint National Committee (JNC) VII [21]. Details of coexistent medical illnesses were collected from the subjects' report of diagnoses as indicated by their doctors and medical charts.

A specialist in internal medicine determined the documentation of diagnoses, and assessments/ measurements for completion of final diagnoses, including heart disease, stroke, and diabetes. The Activity of Daily Living (ADL) is a measure of dependency on others for physical needs and functional status [22]. It has 14 items which comprise two scales: the physical self-maintenance scale (PSMS) (range 0-24) and instrumental activities of daily life scale (IADL) (range 0-32).

PSMS assesses the following six items: bathing, dressing, toileting, grooming, physical ambulation, and feeding. IADL assesses the ability to use telephones and public transportation, to go shopping, to prepare food, and the ability to carry out other daily tasks such as housekeeping, laundry, handling own medications, and finances. The higher the score, the less capable the subject is at performing everyday tasks. Findings with these measures in elderly people, including reliability and validity data, have been published elsewhere [23]. Body mass index $\left(\mathrm{kg} / \mathrm{m}^{2}\right)$ was calculated as body weight (kilograms) divided by height (meters squared). Blood samples were collected after an overnight fast, and analyzed for hemoglobin, albumin, triacylglycerol, total cholesterol, low-density lipoprotein cholesterol and high-density lipoprotein cholesterol by standard laboratory techniques.

\section{Statistical analyses}

Continuous data were expressed as mean \pm standard deviation (SD). Categorical data were expressed as a percentage. Differences in baseline characteristics according to category of eGFR ( $<60$, and $>60 \mathrm{ml}$ / min per $1.73 \mathrm{~m}^{2}$ ) were compared using unpaired Student's $t$ tests for continuous data, and chi-square tests/Fisher's exact tests for categorical data. Multivariable logistic regression was used to determine the association (expressed as odds ratio [OR] and 95\% confidence interval [CI]) between kidney function and cognitive impairment. In the cross-sectional analysis, the predictor variable was CKD (categorical variable) or eGFR (continuous variable), and the outcome was cognitive impairment. In model 1, we controlled for age and gender; in model 2, these variables including stroke, diabetes, triacylglycerol, and ADL score, which are 


\section{Kidney \\ Blood Pressure Research}

Wang/Liu/Cai/Jiang/Liu/Lin: Kidney Function and Cognitive Impairment with Untreated Hypertension in Elderly

Table 1. Baseline characteristics according to estimated GFR in an 80-year-old population with hypertension

\begin{tabular}{|c|c|c|c|c|}
\hline \multirow[t]{2}{*}{ Characteristic } & \multicolumn{2}{|c|}{ eGFR (ml/min per $\left.1.73 \mathrm{~m}^{2}\right)$} & \multirow{2}{*}{$\begin{array}{c}\text { Total } \\
(\mathrm{n}=395)\end{array}$} & \multirow[t]{2}{*}{$P$ Value } \\
\hline & $\geq 60(n=336)$ & $<60(\mathrm{n}=59)$ & & \\
\hline SBP (mmHg) & $154.5 \pm 16.4$ & $155.5 \pm 16.2$ & $154.6 \pm 16.4$ & 0.661 \\
\hline DBP (mmHg) & $76.4 \pm 11.7$ & $76.5 \pm 12.1$ & $76.4 \pm 11.7$ & 0.967 \\
\hline Serum creatinine (mmol/L) & $74.9 \pm 12.5$ & $111.6 \pm 17.3$ & $80.4 \pm 18.7$ & $<0.001$ \\
\hline eGFR (ml/min per $1.73 \mathrm{~m} 2)$ & $84.2 \pm 18.7$ & $50.2 \pm 7.8$ & $79.1 \pm 21.3$ & $<0.001$ \\
\hline Cognitive impairment (\%) & 58.2 & 12.9 & 100 & 0.005 \\
\hline Scores on MMSE & $17.8 \pm 6.0$ & $16.1 \pm 5.6$ & $17.6 \pm 6.0$ & 0.049 \\
\hline Age (years) & $82.9 \pm 2.6$ & $83.9 \pm 2.4$ & $83.0 \pm 2.6$ & 0.006 \\
\hline Female $(\%)$ & 58.7 & 11.1 & 100 & 0.393 \\
\hline Educational levels (\%) & 85.1 & 14.9 & 100 & 0.514 \\
\hline Illiteracy & 77.5 & 13.2 & & \\
\hline Primary school & 5.6 & 1.3 & & \\
\hline Middle school & 1.8 & 0.3 & & \\
\hline High school & 0.3 & 0.3 & & \\
\hline Live alone (\%) & 6.6 & 1.0 & 100 & 0.798 \\
\hline Smoking (\%) & 8.4 & 1.3 & 100 & 0.746 \\
\hline Heart disease (\%) & 3.0 & 1.0 & 100 & 0.276 \\
\hline Stroke (\%) & 0.5 & 1.0 & 100 & 0.055 \\
\hline Diabetes (\%) & 4.6 & 1.8 & 100 & 0.058 \\
\hline Scores on ADL & $17.8 \pm 6.3$ & $22.1 \pm 8.5$ & $18.4 \pm 6.8$ & $<0.001$ \\
\hline Body mass index $(\mathrm{kg} / \mathrm{m} 2)$ & $19.9 \pm 2.9$ & $20.0 \pm 2.7$ & $20.0 \pm 2.9$ & 0.857 \\
\hline Hemoglobin (g/L) & $126.4 \pm 14.6$ & $124.2 \pm 17.7$ & $126.1 \pm 15.1$ & 0.299 \\
\hline Fasting blood glucose & $4.45 \pm 1.28$ & $4.56 \pm 1.32$ & $4.41 \pm 1.39$ & 0.553 \\
\hline Albumin (g/L) & $43.0 \pm 3.3$ & $42.8 \pm 2.5$ & $43.0 \pm 3.2$ & 0.621 \\
\hline Triglycerides (mmol/L) & $4.4 \pm 0.7$ & $4.3 \pm 0.7$ & $4.4 \pm 0.7$ & 0.059 \\
\hline Total cholesterol (mmol/L) & $1.4 \pm 0.7$ & $1.6 \pm 0.8$ & $1.4 \pm 0.7$ & 0.683 \\
\hline LDL (mmol/L) & $2.5 \pm 0.5$ & $2.5 \pm 0.5$ & $2.5 \pm 0.5$ & 0.697 \\
\hline HDL (mmol/L) & $1.9 \pm 0.9$ & $2.0 \pm 1.1$ & $1.9 \pm 0.9$ & 0.350 \\
\hline
\end{tabular}

eGFR, estimated glomerular filtration rate; SBP, systolic blood pressure; DBP, diastolic blood pressure; MMSE, Mini-Mental State Examination; ADL, Activity of Daily Living; LDL, low-density lipoprotein cholesterol; HDL, high-density lipoprotein cholesterol. $\mathrm{P}$ value presents the statistical differences of baseline characteristics beween patients with chronic kidney disease and without chronic kidney disease.

not significant, but close to 0.05 , were also adjusted. P less than 0.05 is considered statistically significant. Statistical analysis was performed with SPSS software package for Windows version 16.0 (SPSS Institute Inc., Chicago, USA).

\section{Results}

395 participants were recruited into the cross-sectional study. Of these participants, 276 were female and had a mean age of $83.0 \pm 2.6$ (SD) years; 358 (90.7\%) of all participants did not receive any formal education. The mean (SD) cognitive function score for the sample was 17.6 (6.0) and the prevalence of cognitive impairment was 71.1\%. The mean (SD) eGFR value was 79.1(21.3) and CKD was present in $14.9 \%(n=59)$ of the sample. Compared with participants without CKD, participants with CKD were older, had higher scores on ADL, and lower scores on MMSE (Table 1). 


\section{Kidney Blood Pressure Research}

Wang/Liu/Cai/Jiang/Liu/Lin: Kidney Function and Cognitive Impairment with Untreated Hypertension in Elderly

Table 2. Odds ratio for cognitive impairment by chronic kidney disease and eGFR in an 80-year-old population with hypertension

\begin{tabular}{ccccc}
\hline & \multicolumn{2}{c}{ CKD } & \multicolumn{2}{c}{ eGFR } \\
\cline { 2 - 5 } & OR, 95\%CI & P & OR, 95\%CI & P \\
\hline Model 1 & $3.367(1.339,8.466)$ & 0.010 & $0.986(0.974,0.997)$ & 0.013 \\
Model 2 & $2.764(1.069,7.151)$ & 0.036 & $0.988(0.977,0.999)$ & 0.038 \\
\hline
\end{tabular}

CKD, chronic kidney disease; eGFR, estimated glomerular filtration rate. Model 1, adjustment for age and gender. Model 2 contains the same variables as Model $1+$ (diabetes, stroke, triglycerides, and ADL scores)

In unadjusted analyses, CKD was associated with a significant increased prevalence of cognitive impairment. The association remained significant after additional adjustment for demographic characteristics (model 1: OR 3.367; 95\% CI, 1.339 to 8.466), and ADL scores, diabetes, stroke, and triglycerides (model 2: OR 2.764; 95\% CI, 1.069 to 7.151). When eGFR as a predictor variable entered into the model, the result remained significant in the two models (model 1: OR 0.986; 95\% CI, 0.974 to 0.997; model 2: OR 0.988; $95 \% \mathrm{CI}, 0.977$ to 0.999 ) after adjusting for the same confounding factors (Table 2).

\section{Discussion}

In the cross-sectional study of 395 untreated hypertensive persons aged 80 or over in China, we found an association between CKD/eGFR and cognitive impairment, which was independent of potential confounding factors including age, gender, stroke, diabetes, ADL scores, and triacylglycerol.

These findings support the findings observed by several published cross-sectional studies. In a single-center study, 160 subjects with CKD had worse performance on tests of executive function and verbal memory compared with published norms [24]. Furthermore, in the Reasons for Geographic and Racial Difference in Stroke (REGARDS Study), CKD was associated with an increased prevalence of cognitive impairment independent of confounding factors, and for subjects with a eGFR less than $60 \mathrm{~mL} / \mathrm{min} / 1.73 \mathrm{~m}^{2}$, each $10 \mathrm{~mL} / \mathrm{min} / 1.73 \mathrm{~m}^{2}$ decrease was associated with an $11 \%$ increased prevalence of cognitive impairment [9]. Other retrospective studies confirmed and extended this relationship to those with mildly reduced eGFR as measured according to neurophysiological tests and comprehensive testing in a particular domain for cognitive function, suggesting that even small reductions in kidney function are associated with clinically significant consequences for cognitive function $[7,8]$. Recently, several longitudinal studies $[3,16]$, in particular a study from China [6], have indicated that reduced kidney function is associated with cognitive decline and the relationship is independent of potential confounders, including urinary albumin excretion. Compared with the studies mentioned above, the current study only included hypertensive patients, and also confirmed the association between CKD and cognitive impairment in hypertensive patients, even after controlling blood pressure. This suggests that the treatment focusing on reduced kidney function should be considered among aged hypertensive patients with impaired cognition, although only a small percentage of patients with hypertension will develop CKD [25]. To the best of our knowledge, few studies have explored an association between CKD or eGFR and cognitive impairment in persons aged 80 or over. Therefore, our study also extends the findings of previous studies, because the average age of our study is older than that of any previous study. In addition, our study included untreated hypertensive patients rather than treated hypertensive patients [3], which will remove possible confounds of antihypertensive drugs.

There are several mechanisms that might link CKD with cognitive impairment. First, many traditional risk factors for cognitive impairment, such as diabetes, hyperlipemia, heart disease, and stroke often co-existed with CKD, which may lead to cognitive impairment [26-28]. Second, CKD is associated with white matter hyperdensities and silent infarctions 


\section{Kidney \\ Blood Pressure Research}

Kidney Blood Press Res 2016;41:70-77

\begin{tabular}{l|l}
\hline DOI: $10.1159 / 000368550$ & (c) 2016 S. Karger AG, Basel
\end{tabular}

Published online: February 14, 2016

www.karger.com/kbr

Wang/Liu/Cai/Jiang/Liu/Lin: Kidney Function and Cognitive Impairment with Untreated Hypertension in Elderly

suggesting that subclinical cerebrovascular disease underlies the association of kidney function and cognition [29]. Third, other abnormalities in CKD patients including anemia, hypoproteinemia, sleep disturbance, depression, inflammation, and oxidative stress could have an important role in the development and progression of cognitive impairment in hypertension [26, 30]. However, many established risk factors for cognitive impairment in the younger old, such as hypertension, metabolic syndrome and hyperlipoidemia, may be less important in the oldest old $[31,32]$. Authors speculated that persons who reach the age of 90 or over, despite having cardiovascular diseases, are survivors who are relatively less susceptible to cognitive impairment. In other words, long-lived subjects might have fewer risk factors for cerebrovascular disease than do normal (less long-lived) elderly persons. Our study included subjects aged below 90 , thus this explanation may not be suitable for the relationship between CKD and cognitive impairment.

Our study provides increased evidence for the association between kidney function and cognition among hypertensive patients aged 80 or over. However, there are some limitations that deserve mention. First, our study is cross-sectional and thus does not allow us to determine causal directions between cognitive impairment and CKD. Second, kidney function was estimated by applying a creatinine-based equation (CMDRD), which may be inaccurate for evaluation of kidney function among the oldest old people, because it depends to some extent on muscle mass and nutritional status. This can lead to overestimation of kidney function in elderly people with malnutrition, as malnutrition often increases with ageing. Third, using an MMSE score of $<24$ is considered to be less sensitive for mild cognitive impairment than other tests, including Modified MMSE and Cognitive Capacity Screening Examination, even though diagnosis of cognitive impairment using this cut-off has been shown to be $80-90 \%$ sensitive and $80-100 \%$ specific compared with DSMMD- IV. However, the type of variation would bias our results toward null findings; it is therefore possible that we underestimated the true relationship. Fourth, our study lacked adjustment for other potential confounders, such as inflammatory markers, malnutrition status, heredity, and other sociodemographic factors, which may explain these results.

\section{Conclusion}

In conclusion, both CKD and eGFR were associated with cognitive impairment among hypertensive patients aged 80 or over in China. It could imply that measures of kidney function should be included in the assessment of cognitive impairment risk factors in hypertensive patients. In addition, given the high prevalence of CKD and cognitive impairment in hypertensive patients aged 80 or over, future clinical trials to improve cognition should consider targeting this high-risk population.

\section{Disclosure Statement}

The authors declare that they have no competing interests.

\section{Acknowledgements}

This work was supported by grants from the Sichuan health department (No 110029). 


\section{Kidney \\ Blood Pressure Research}

Kidney Blood Press Res 2016;41:70-77

\begin{tabular}{l|l}
\hline DOI: $10.1159 / 000368550$ & (C) 2016 S. Karger AG, Basel
\end{tabular}

Published online: February 14, 2016

www.karger.com/kbr

Wang/Liu/Cai/Jiang/Liu/Lin: Kidney Function and Cognitive Impairment with Untreated Hypertension in Elderly

\section{References}

1 Nitta K, Okada K, Yanai M, Takahashi S: Aging and chronic kidney disease. Kidney Blood Press Res 2013;38:109-120.

2 Murray AM: Cognitive impairment in the aging dialysis and chronic kidney disease populations: an occult burden. Adv Chronic Kidney Dis 2008;15:123-132.

3 Peters R, Beckett N, Forette F, Tuomilehto J, Ritchie C, Walton I, Waldman A, Clarke R, Poulter R, Fletcher A, Bulpitt C: Vascular risk factors and cognitive function among 3763 participants in the Hypertension in the Very Elderly Trial (HYVET): a cross-sectional analysis. Int Psychogeriatr 2009;21:359-368.

4 Zhang YP, Zuo XC, Huang ZJ, Kuang ZM, Lu MG, Duan DD, Yuan H: The impact of blood pressure on kidney function in the elderly: a cross-sectional study. Kidney Blood Press Res 2013;38:205-216.

5 Sierra C, Domenech M, Camafort M, Coca A: Hypertension and mild cognitive impairment. Curr Hypertens Rep 2012;14:548-555.

6 Wang F, Zhang L, Liu L, Wang H: Level of kidney function correlates with cognitive decline. Am J Nephrol 2010;32:117-121.

7 Kurella M, Yaffe K, Shlipak MG, Wenger NK, Chertow GM: Chronic kidney disease and cognitive impairment in menopausal women. Am J Kidney Dis 2005;45:66-76.

8 Etgen T, Sander D, Chonchol M, Briesenick C, Poppert H, Förstl H, Bickel H: Chronic kidney disease is associated with incident cognitive impairment in the elderly: the INVADE study. Nephrol Dial Transplant 2009;24:3144-3150.

9 Kurella M, Wadley V, Yaffe K, McClure LA, Howard G, Go R, Allman RM, Warnock DG, McClellan W: Kidney function and cognitive impairment in US adults: the Reasons for Geographic and Racial Differences in Stroke (REGARDS) Study. Am J Kidney Dis 2008;52:227-234.

10 Darsie B, Shlipak MG, Sarnak MJ, Katz R, Fitzpatrick AL, Odden MC: Kidney function and cognitive health in older adults: the Cardiovascular Health Study. Am J Epidemiol 2014;180:68-75.

11 Basile J: Chronic kidney disease: It's time to recognize its presence in our patients with hypertension. J Clin Hypertens 2004;6:548-552.

12 da Matta SM, Janaina Matos M, Kummer AM, Barbosa IG, Teixeira AL, Silva AC: Cognitive alterations in chronic kidney disease: an update. J Bras Nefrol 2014;36:241-245.

13 Egbi OG, Ogunrin O, Oviasu E: Prevalence and determinants of cognitive impairment in patients with chronic kidney disease: a cross-sectional study in Benin City, Nigeria. Ann Afr Med 2015;14:75-81.

14 Slinin Y, Paudel ML, Ishani A, Taylor BC, Yaffe K, Murray AM, Fink HA, Orwoll ES, Cummings SR, BarrettConnor E, Jassal S, Ensrud KE: Kidney function and cognitive performance and decline in older men. J Am Geriatr Soc 2008;56:2082-2088.

15 Ji-Rong Y, Bi-Rong D, Chang-Quang H, Hong-Mei W, Yan-Ling Z, Qing-Xiu L, Jue-Ling D, Bing-You W, QiYuan Y: Cognitive impairment and depression among Chinese nonagenarians/centenarians. Am J Geriatr Psychiatry 2010;18:297-304.

16 Peters R, Poulter R, Beckett N, Forette F, Fagard R, Potter J, Swift C, Anderson C, Fletcher A, Bulpitt CJ: Cardiovascular and biochemical risk factors for incident dementia in the Hypertension in the Very Elderly Trial. J Hypertens 2009;27:2055-2062.

17 Ma YC, Zuo L, Chen JH, Luo Q, Yu XQ, Li Y, Xu JS, Huang SM, Wang LN, Huang W, Wang M, Xu GB, Wang HY: Modified glomerular filtration rate estimating equation for Chinese patients with chronic kidney disease. J Am Soc Nephrol 2006;17:2937-2944.

18 Zhang MY, Katzman R, Salmon D, Jin H, Cai G, Wang Z, Qu G, Grant I, Yu E, Levy P, Klauber MR, Liu WT: The prevalence of dementia and Alzheimer's disease in Shanghai, China: impact of age, gender, and education. Ann Neurol 1990;27:428-437.

19 Tombaugh TN, McIntyre NJ: The Mini-Mental State exam: a comprehensive review. J Am Geriatr Soc 1992;40:922-993.

20 Zhang MY: Comparison of several screening instruments for dementia. Zhonghua Shen Jing Jing Shen Ke Za Zhi 1991;24:194-196.

21 Cuddy ML: Treatment of hypertension: guidelines from JNC 7 (the seventh report of the Joint National Committee on Prevention, Detection, Evaluation, and Treatment of High Blood Pressure 1). J Pract Nurs 2005;55:17-21. 


\section{Kidney \\ Blood Pressure Research}

Kidney Blood Press Res 2016;41:70-77

DOI: 10.1159/000368550

(C) 2016 S. Karger AG, Basel

Published online: February 14, 2016

www.karger.com/kbr Hypertension in Elderly

22 Lawton MP, Brody EM: Assessment of older people: self-maintaining and instrumental activities of daily living. Gerontologist 1969;9:179-186.

23 Green CR, Mohs RC, Schmeidler J, Arvan M, Davis KL: Functional decline in Alzheimer's disease: a longitudinal study. J Am Geriatric Soc 1993;41:654-661.

24 Kurella M, Chertow GM, Luan J, Yaffe K: Cognitive impairment in chronic kidney disease. J Am Geriatr Soc 2004;52:1863-1869.

25 Freedman BI, Sedor JR: Hypertension-associated kidney disease: perhaps no more. J Am Soc Nephrol 2008;19:2047-2051.

26 Elias MF, Dore GA, Davey A: kidney disease and cognitive function. Contrib Nephrol 2013;17:42-57.

27 Koren-Morag N, Goldbourt U, Tanne D: Renal dysfunction and risk of ischemic stroke or TIA in patients with cardiovascular disease. Neurology 2006;67:224-228.

28 Tokmakova MP, Skali H, Kenchaiah S, Braunwald E, Rouleau JL, Packer M, Chertow GM, Moyé LA, Pfeffer MA, Solomon SD: Chronic kidney disease, cardiovascular risk, and response to angiotensin-converting enzyme inhibition after myocardial infarction: the Survival and Ventricular Enlargement (SAVE) Study. Circulation 2004;110:3667-3673.

29 Kobayashi M, Hirawa N, Yatsu K, Kobayashi Y, Yamamoto Y, Saka S, Andoh D, Toya Y, Yasuda G, Umemura S: Relationship between silent brain infarction and chronic kidney disease. Nephrol Dial Transplant 2009;24:201-207.

30 Gasecki D, Kwarciany M, Nyka W, Narkiewicz K: Hypertension, brain damage and cognitive decline. Curr Hypertens Rep 2013;15:547-558.

31 van den Berg E, Biessels GJ, de Craen AJ, Gussekloo J, Westendorp RG: The metabolic syndrome is associated with decelerated cognitive decline in the oldest old. Neurology 2007;69:979-985.

32 Huang CQ, Dong BR, Zhang YL, Wu HM, Liu QX, Flaherty JH: cognitive impairment and hypertension among Chinese nonagenarians and centenarians. Hypertens Res 2009;32:554-558. 